\title{
Effect of melatonin on the functional recovery from experimental traumatic compression of the spinal cord
}

\author{
A. Schiaveto-de-Souza ${ }^{1}$, C.A. da-Silva ${ }^{2}$, H.L.A. Defino ${ }^{3}$ and E.A. Del Bel ${ }^{2}$ \\ ${ }^{1}$ Departamento de Morfofisiologia, Universidade Federal do Mato Grosso do Sul, Campo Grande, MS, Brasil \\ ${ }^{2}$ Departamento de Morfologia, Estomatologia e Fisiologia, Faculdade de Odontologia de Ribeirão Preto, Universidade de São Paulo, \\ Ribeirão Preto, SP, Brasil \\ ${ }^{3}$ Departamento de Orthopedia e Traumatologia, Faculdade de Medicina de Ribeirão Preto, Universidade de São Paulo, \\ Ribeirão Preto, SP, Brasil
}

\begin{abstract}
Spinal cord injury is an extremely severe condition with no available effective therapies. We examined the effect of melatonin on traumatic compression of the spinal cord. Sixty male adult Wistar rats were divided into three groups: sham-operated animals and animals with 35 and $50 \%$ spinal cord compression with a polycarbonate rod spacer. Each group was divided into two subgroups, each receiving an injection of vehicle or melatonin $(2.5 \mathrm{mg} / \mathrm{kg}$, intraperitoneal) $5 \mathrm{~min}$ prior to and 1, 2, 3, and $4 \mathrm{~h}$ after injury. Functional recovery was monitored weekly by the open-field test, the Basso, Beattie and Bresnahan locomotor scale and the inclined plane test. Histological changes of the spinal cord were examined 35 days after injury. Motor scores were progressively lower as spacer size increased according to the motor scale and inclined plane test evaluation at all times of assessment. The results of the two tests were correlated. The open-field test presented similar results with a less pronounced difference between the 35 and $50 \%$ compression groups. The injured groups presented functional recovery that was more evident in the first and second weeks. Animals receiving melatonin treatment presented more pronounced functional recovery than vehicle-treated animals as measured by the motor scale or inclined plane. NADPH-d histochemistry revealed integrity of the spinal cord thoracic segment in sham-operated animals and confirmed the severity of the lesion after spinal cord narrowing. The results obtained after experimental compression of the spinal cord support the hypothesis that melatonin may be considered for use in clinical practice because of its protective effect on the secondary wave of neuronal death following the primary wave after spinal cord injury.
\end{abstract}

Key words: Spinal cord injury; Melatonin; Functional recovery

\section{Introduction}

Spinal cord injury $(\mathrm{SCl})$ results in the loss of function below the lesion. The compression of the spinal cord that follows vertebral displacement and edema is considered to be a very frequent cause of traumatic spinal cord lesion. Secondary injury following the primary impact includes a number of biochemical and cellular alterations, localized edema, hemorrhage, thrombosis, vasospasm, and loss of vasculature autoregulation (1). Primary traumatic mechanical injury to the spinal cord causes the death of a number of neurons that cannot be recovered or regenerated. Some neurons continue to die for hours after traumatic $\mathrm{SCI}(2,3)$. This secondary neuronal death may be caused by substances released from cells in response to the primary injury. Over a period of minutes to hours the affected area undergoes complex processes that give rise to secondary damage. The initial procedures are thought to be decisive for the evolution of the events that ensue during the evolution of the injury (1).

Several studies have revealed that early interventions can produce positive effects regarding the consequences of secondary injury $(2,3)$. Growing evidence has shown that melatonin (5-methoxy- $\mathrm{N}$-acetyltryptamine) is a neuroprotective compound. Melatonin is a neurohormone synthesized from L-tryptophan in the pineal gland, retina and gastrointestinal tract, released into the blood with a circadian rhythm that peaks during the night in mammals (4). Its rhythmic production by the pineal gland was described in human beings over 20 years ago. However,

Correspondence: E.A. Del Bel, Departamento de Morfologia, Estomatologia e Fisiologia, Faculdade de Odontologia de Ribeirão Preto, Universidade de São Paulo, 14049-904 Ribeirão Preto, SP, Brasil. Fax: +55-16-3602-4047. E-mail: eadelbel@usp.br 
melatonin production is not confined exclusively to the pineal gland, but other tissues including retina, Harderian glands, gut, ovary, testes, bone marrow, and lens also produce it. Melatonin is rapidly absorbed because of its lipophilic nature, and readily crosses the blood-brain barrier (5). Reduction of endogenous melatonin after pinealectomy makes rats more vulnerable to trauma and exogenous melatonin administration has an important neuroprotective effect. The spinal cord is particularly vulnerable to free radical oxidation because of its high lipid content. Pharmacological doses of melatonin do not produce in vivo toxic effects and seem to support some features of the antioxidant defense systems (5).

The question of the neuroprotective effect of melatonin after spinal cord injury is controversial. After compression ischemic-induced $\mathrm{SCl}$, Fujimoto et al. (6) showed that melatonin reduced the occurrence of neutrophilinduced lipid peroxidation, and that it facilitated recovery of the damaged spinal cord. Topsakal et al. (7) showed that melatonin applied early after $\mathrm{SCl}$ not only reduced lipid peroxidation but also prevented the decrease of superoxide dismutase and glutathione peroxidase, components of the endogenous antioxidant system. Protection of the spinal cord against lipid peroxidation by melatonin was similar to that provided by methylprednisolone (3). However, Guízar-Sahagún et al. (8) showed no pharmacological neuroprotection in melatonin-treated rats in an animal model of spinal surgery-induced neurological damage (no differences in their locomotor performance or in the amount of spared cord tissue).

The purpose of the present study was to further investigate the neuroprotective effect of melatonin. We examined the effect of melatonin on neurobehavioral impairment produced by traumatic compression of the spinal cord in rats. The tested hypothesis was that the use of melatonin immediately after spinal cord compression could improve the rate and extent of motor recuperation of the animals. The results reported here support the benefit of exogenous melatonin as a therapeutic intervention for spinal cord injury induced by compression.

\section{Material and Methods}

\section{Animals}

Male Wistar rats $(250-300 \mathrm{~g}, \mathrm{n}=60)$ were kept in a temperature-controlled room $\left(23^{\circ} \mathrm{C}\right)$ with a 12-h light/dark cycle (lights on at 7:00 am) and with free access to water and food. Experiments were performed between 8:00 and 12:00 am. The experiments were carried out according to the guidelines of the Brazilian Society of Neuroscience and Behavior that comply with the National Institutes of Health Guide for care and use of laboratory animals. A white noise generator provided a constant background noise and the apparatus was cleaned and dried before each session with $70 \%$ ethanol to minimize olfactory cues.

\section{Spinal cord compression}

Spacers (manufactured using a polycarbonate rod) were designed to induce spinal cord compression, as described by Dimar et al. (9). They were manufactured in such a way as to decrease the rat's average spinal canal diameter by $35 \%$ (0.78-mm thick spacer) and 50\% (1.11$\mathrm{mm}$ thick spacer). The spacers were precisely measured to ensure correct dimensions using microcalipers (9).

\section{Surgical procedure}

All surgical techniques were performed under aseptic conditions. Animals were anesthetized with an intraperitoneal (ip) injection of $250 \mathrm{mg} / \mathrm{kg}$ 2,2,2-tribromoethanol (Aldrich Chemical Company, USA), followed by subcutaneous administration of penicillin. After shaving the skin, animals were kept in a stereotaxic apparatus (David Kopf, USA) and a posterior incision in the skin and underlying muscles was made in the mid-thoracic region (10). Muscles were retracted and a mid-thoracic total laminectomy was performed at the level of the T10 vertebra. When required, the dura mater was exposed. According to the experimental group, spacers of different sizes were introduced under the T9 lamina in the dorsal epidural space to promote spinal cord compression (Figure 1), while in the sham-operated group no spacer was introduced and the spinal cord was free of compression. In the compression groups, the spacers were kept under the T9 lamina until the end of the experiment.

After the surgical procedure, the paraspinal fascia, muscle and skin were closed with 4-0 nylon suture (Ethicon, Brazil). The animals were placed in a warming chamber and body temperature was maintained at approximately $37^{\circ} \mathrm{C}$ until they were fully awake, at which time they were returned to their home cages, 2 or 3 rats per cage. Postoperative care included regular bladder expression.

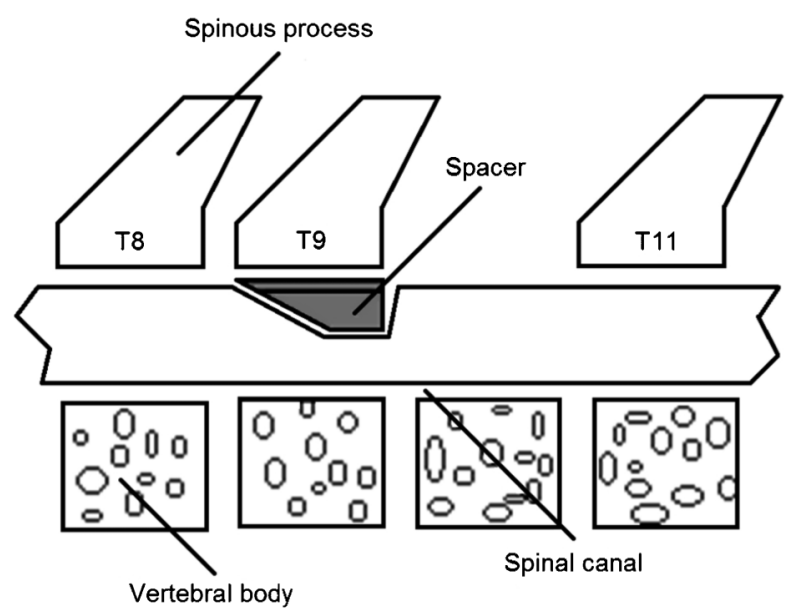

Figure 1. Schematic overview of placement of the spacer under the T9 lamina to promote spinal cord compression. 


\section{Behavioral evaluation methods}

Behavioral parameters were recorded preoperatively and $1,7,14,21,28$, and 35 days after the experimental procedure. The same animals were tested in the open arena and evaluated by the Basso, Beattie and Bresnahan (BBB) locomotor score and inclined plane test.

Open-field test. To assess their exploratory behavior, rats were tested in an open field consisting of a circular wooden box ( $72 \mathrm{~cm}$ in diameter) surrounded by $49-\mathrm{cm}$ high transparent walls with an open top. The floor was subdivided into 12 equal size fields. In this test, each rat was gently placed in the center of the open field and handoperated counters and stopwatches were used to score. Two different behavioral measures of spontaneous activity were used: locomotion or exploratory movement, measured as the number of floor units entered with the four paws, and rearing frequency, as the number of times the animal stood on its hind limbs. The two different parameters of spontaneous behavior used were empirically found to be best for measurements of individual and group behaviors. Exploratory measures are necessary since stationary activity may not accurately reflect the groups' exploratory behavior in the field. We evaluated changes in each parameter individually.

BBB locomotor scale. The BBB scale, elaborated by Basso et al. (11), is used to analyze specific components of functional behavior such as limb movement, trunk position/instability, paw placement/position, stepping, coordination, toe clearance, and tail position. The scores range from 0 (no detectable hind limb movement) to 21 (normal locomotion) and have been extensively described elsewhere (11). The locomotor activity of individual animals was evaluated in the circular wooden open arena for $4 \mathrm{~min}$ by 1 examiner observing the rat. The observer did not touch the animal in the open field. A rating sheet described by Basso et al. (11) was used for individual animal recording.

Inclined plane test. The inclined plane test was performed as described by Rivlin and Tator (12). In this test the rat was placed on a mat in such a way that its body axis was perpendicular to the axis of an inclined board, which could be adjusted to provide a slope of varying grade. The angle of the inclined plane was the maximum angle of the plane at which a rat could maintain itself for at least $5 \mathrm{~s}$.

\section{Histological analysis}

After 35 days the animals ( $n=10$ per group) were deeply anesthetized with a lethal ip injection of $25 \%$ urethane, and sacrificed by intracardiac perfusion with $0.01 \mathrm{M}$ PBS $(200 \mathrm{~mL}, \mathrm{pH} 7.4)$ followed by $4 \%$ paraformaldehyde in $0.1 \mathrm{M}$ phosphate buffer $(200 \mathrm{~mL}, \mathrm{pH} 7.4)$. The spinal cord was rapidly removed and soaked in the same fixative solution for $2 \mathrm{~h}\left(4^{\circ} \mathrm{C}\right)$ and then cryoprotected by overnight soaking in $30 \%$ sucrose/phosphate buffer. Thoracic segments of the spinal cord were frozen in isopentane $\left(-40^{\circ} \mathrm{C}\right.$, Sigma, USA) and stored at $-70^{\circ} \mathrm{C}$ until the time for the histochemical procedures.

Histochemistry for NADPH-diaphorase. Forty-micrometer serial sections were cut with a cryostat (Leica, Germany) and neuroanatomical sites were identified using the Paxinos and Watson atlas (13). NADPHdiaphorase (NADPH-d) activity was measured by incubating free-floating sections in $0.1 \mathrm{M}$ phosphate buffer, $\mathrm{pH}$ 7.4, containing 0.3\% Triton-X 100 (Sigma), $0.1 \mathrm{mg} / \mathrm{mL}$ Nitroblue tratrazolium (Sigma) and $0.1 \mathrm{mg} / \mathrm{mL} \beta-\mathrm{NADPH}$, at $37^{\circ} \mathrm{C}$ for $90 \mathrm{~min}$ in the dark. The sections were dipped for $10 \mathrm{~min}$ in phosphate buffer, dried, rinsed in distilled water, and mounted for microscopic observation (14). Slices from about $8 \mathrm{~mm}$ of the spinal cords were examined with a microscope linked to a computerassisted image-processing system through a video camera. For cell density quantification, the spinal cord was divided into five regions: 1) the superficial lamina (laminae I and II), 2) the nucleus proprius (laminae III and IV), 3) the intermediolateral cell column, 4) the region around the central canal (lamina $X$ ), and 5) the ventra horn (laminae VIII and IX). NADPH-d-positive neuron density was measured in a blind manner in two regions of interest, i.e., rostral $(0.0-3.9 \mathrm{~mm})$ and caudal $(0.0-$ $3.9 \mathrm{~mm}$ ) spinal segments beyond the narrowing site.

\section{Experimental groups}

The experimental groups ( $n=10$ per group) were formed according to the degree of spinal canal narrowing (sham-operated, 35\% compression and 50\% compression) and the administration of melatonin or vehicle, for a total of 6 experimental groups: SO-V (sham-operated + vehicle), $35 \%-V$ (narrowing of $35 \%+$ vehicle), $50 \%-V$ (narrowing of $50 \%+$ vehicle), SO-M (sham-operated + melatonin), 35\%-M (narrowing of 35\% + melatonin), and $50 \%-\mathrm{M}$ (narrowing of $50 \%+$ melatonin). Melatonin (Sigma, $2.5 \mathrm{mg} / \mathrm{kg}$ dissolved in absolute ethanol-saline, $5: 95, \mathrm{v} / \mathrm{v}$ ) or vehicle was administered ip at $5 \mathrm{~min}$ prior to and $1,2,3$, and $4 \mathrm{~h}$ after injury. Dosage was in accordance with the pharmacokinetic properties of melatonin (15) and as described by Fujimoto et al. (6).

\section{Statistical analysis}

The behavioral data were submitted to repeated measure multivariate analysis of variance (MANOVA) followed by one-way ANOVA and the Duncan post hoc test in case of significant treatment $x$ time interaction. The Student $t$-test was also used to compare drug treatments. The same tests were used to assess the effects of compression and of melatonin treatment on NADPH-dpositive neurons of different gray matter Rexed laminae. Pearson's correlation coefficient was employed to compare the behavioral tests. The level of significance was set at $\mathrm{P}<0.05$. Data are reported in graphs as means \pm $\mathrm{SE}$, with the number of data points indicated for each mean. 


\section{Results}

\section{Open-field test}

Quantitative analysis of ambulation in order to evaluate locomotive ability is presented in Figure 2. A $35 \%$ narrowing of the spinal cord canal resulted in a significant decrease of squares crossed by the animals when compared to the SO-V group at days 1,7 , and 14 after injury. In the $50 \%-\mathrm{V}$ animals, an additional decrease was detected at days $1,7,14$, and 28 , although this decrease was more pronounced in the $50 \%-\mathrm{V}$ group than in the $35 \%-V$ group only on the first day $\left(F_{2,19}\right.$ variation

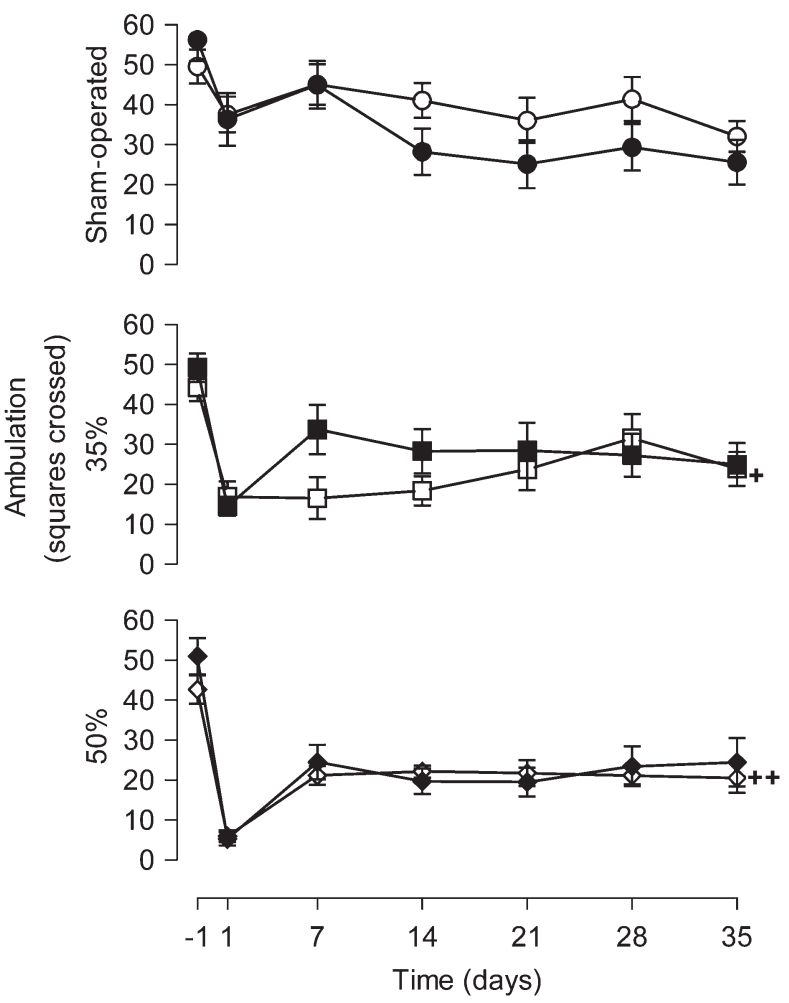

Figure 2. Melatonin treatment did not affect the ambulation of rats. Numbered squares crossed in an open arena were measured $24 \mathrm{~h}$ before $(-1)$ and $1,7,14,21,28$, and 35 days after narrowing of the spinal cord canal. Each point represents the mean \pm SE for 9-11 rats. ${ }^{+} P<0.001$ compared to shamoperated group from vehicle-treated animals, on days 1, 7 and 14 after narrowing of the spinal cord canal (ANOVA with the Duncan post hoc test). ${ }^{++} P<0.001$ compared to sham-operated group from vehicle-treated animals, on days $1,7,14$, and 28 after narrowing of the spinal cord canal and compared to $35 \%$ narrowing from vehicle-treated animals, on the first day after narrowing of the spinal cord canal (ANOVA with the Duncan post hoc test). Melatonin application did not change the number of floor units crossed by the animals of the three groups (MANOVA, $P>0.05) .35 \%=35 \%$ narrowing group; $50 \%=50 \%$ narrowing group. Open symbols $=$ vehicle-treated groups; filled symbols $=$ melatonin-treated groups. from 6.13 to 29.02, $P<0.01$ ). Rearing behavior decreased with time in all groups (time effect: $F_{6,114}=$ 58.42, $\mathrm{P}<0.0001)$. However, this decrease was more pronounced in animals with narrowing of the spinal cord canal at all times post-injury $\left(F_{2,19}\right.$ variation from 17.91 to 69.31, $P<0.0001)$. No difference was observed between the $35 \%-V$ and $50 \%-V$ groups ( $P>0.05$; Figure 3 ).

Melatonin application did not change the number of floor units crossed by the animals of the three groups (drug effect: SO-M, $\mathrm{F}_{1,15}=0.83, \mathrm{P}=0.38 ; 35 \%-\mathrm{M}, \mathrm{F}_{1,13}$ $\left.=0.77, \mathrm{P}=0.40 ; 50 \%-\mathrm{M}, \mathrm{F}_{1,19}=0.26, \mathrm{P}=0.61\right)$. In contrast, the number of rearing events was modified by melatonin in the 35\%-M group (drug effect: $F_{1,13}=5.45$, $P=0.036)$. Animals that received melatonin explored the vertical space more than vehicle-treated ones on days 14
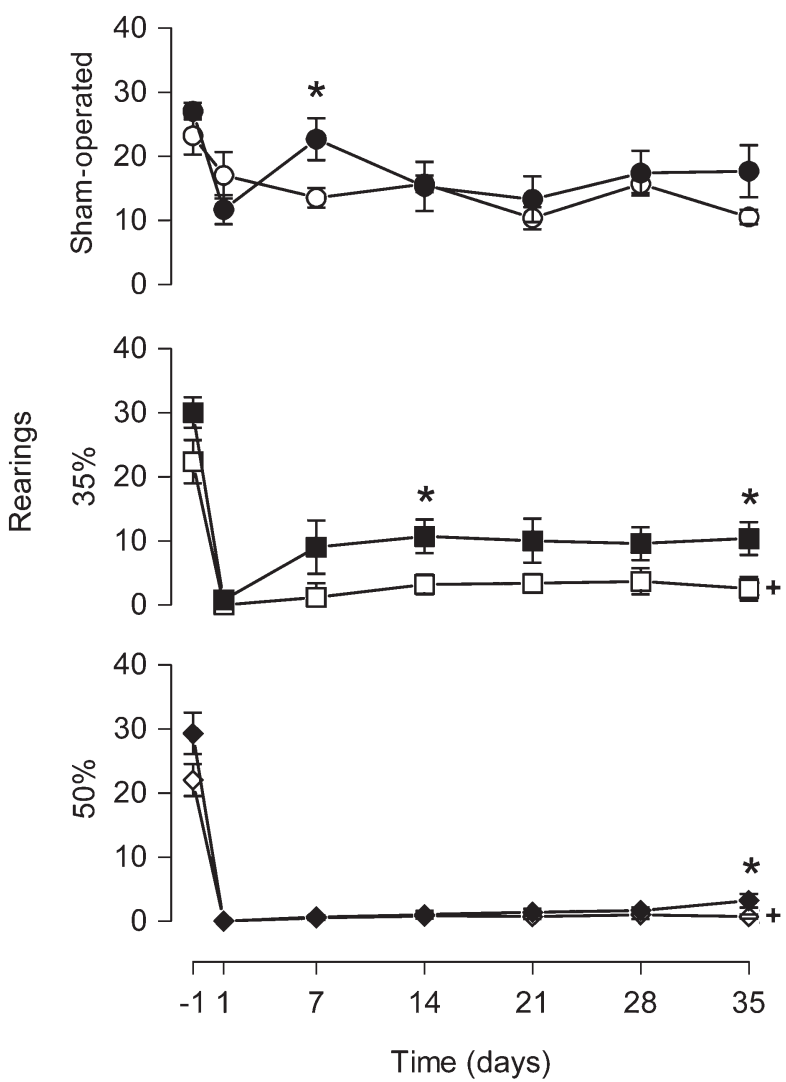

Figure 3. Melatonin treatment increased the rearing behavior Rearing behavior in an open arena was measured $24 \mathrm{~h}$ before $(-1)$ and $1,7,14,21,28$, and 35 days after narrowing of the spinal cord canal. Each point represents the mean \pm SE for 9-11 rats. ${ }^{*} \mathrm{P}<0.05$ compared to the vehicle-treated group (independent Student $t$-test). ${ }^{+} \mathrm{P}<0.0001$ compared to sham-operated group from vehicle-treated animals at all times after narrowing of the spinal cord canal (ANOVA with the Duncan post hoc test). No difference was observed between the $35 \%-\mathrm{V}$ and $50 \%-\mathrm{V}$ groups $(P>0.05) .35 \%=35 \%$ narrowing group; $50 \%=50 \%$ narrowing group. Open symbols $=$ vehicle-treated groups; filled symbols $=$ melatonin-treated groups. 
and 35 after injury ( $\mathrm{t}_{13}$ ranging from -2.35 to $-2.18, \mathrm{P}<$ 0.05). However, in the sham-operated and $50 \%$ groups, melatonin had no effect on rearing behavior (drug effect: sham-operated, $\mathrm{F}_{1,15}=0.72, \mathrm{P}=0.41 ; 50 \%, \mathrm{~F}_{1,19}=$ 3.93, $P=0.06)$.

\section{BBB locomotor score}

After laminectomy, no functional deficit was detected in the animals at any time point of assessment (21 points; Figure 4). Narrowing of the spinal cord canal produced changes in locomotor behavior (narrowing effect: $F_{2,28}=$ $226.09, \mathrm{P}<0.0001$ ). Animals of the $35 \%-\mathrm{V}$ and $50 \%-\mathrm{V}$ groups exhibited significant locomotor impairment at days
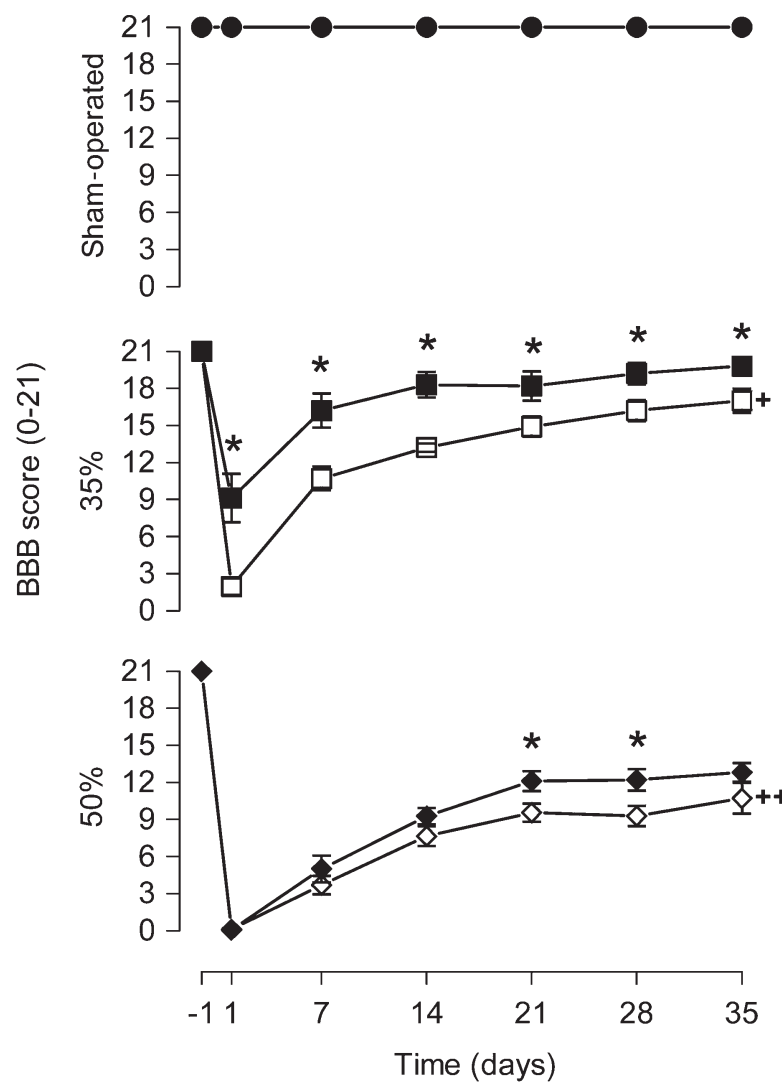

Figure 4. Melatonin treatment improved the BBB score. Results of the Basso, Beattie and Bresnahan (BBB) locomotor scale measured $24 \mathrm{~h}$ before $(-1)$ and $1,714,21,28$, and 35 days after narrowing of the spinal cord canal. Each point represents the mean \pm SE for 9-11 rats. ${ }^{*} P<0.05$ compared to the vehicletreated group (independent Student $t$-test). ${ }^{+} \mathrm{P}<0.0001$ compared to sham-operated group from vehicle-treated animals, at all times after narrowing of the spinal cord canal (ANOVA with the Duncan post hoc test). ${ }^{++} \mathrm{P}<0.0001$ compared to shamoperated group and $35 \%$ narrowing from vehicle-treated animals, at all times after narrowing of the spinal cord canal (ANOVA with the Duncan post hoc test). $35 \%=35 \%$ narrowing group; $50 \%=$ $50 \%$ narrowing group. Open symbols = vehicle-treated groups; filled symbols $=$ melatonin-treated groups .
$1,7,14,21,28$, and 35 after narrowing compared to SO-V ones $\left(F_{2,28}\right.$ ranging from 30.90 to $\left.953.79, P<0.0001\right)$. Rats of the $50 \%-\mathrm{V}$ group showed significantly lower scores than $35 \%-V$ animals at all times analyzed postinjury $(P<0.05)$. On the first day after injury, 35\%-V and $50 \%-\mathrm{V}$ animals presented BBB scores close to 2 . Progressive functional recuperation was observed over time for both the $35 \%-\mathrm{V}$ and $50 \%-\mathrm{V}$ groups $\left(35 \%-\mathrm{V}, \mathrm{F}_{6,54}\right.$ $=86.47, \mathrm{P}<0.0001 ; 50 \%-\mathrm{V}, \mathrm{F}_{6,60}=128.77, \mathrm{P}<$ $0.0001)$. Recuperation was more pronounced in the $35 \%$ group, whose average BBB score increased from 2 on the first day to 17 after 35 days (15 points). The recuperation range was about 10 points in the $50 \%-V$ group.

Melatonin did not alter the locomotor performance of SO-M animals (drug effect: $F_{1,19}=0.00, P=1.00$ ). However, BBB scores for melatonin-treated animals were always higher than the scores for vehicle-treated animals in the $35 \%-\mathrm{M}$ group ( $\mathrm{t}_{17}$ varying from -4.76 to $-2.34, \mathrm{P}<$ $0.04)$. Likewise, the same effect was observed in the $50 \%$ $M$ group on days 21 and 28 (day 21, $\mathrm{t}_{20}=-2.33, \mathrm{P}=0.03$ day $\left.28, t_{20}=-2.42, P=0.025\right)$. While on the first day after injury $35 \%-V$ animals presented BBB scores close to 2, $35 \%-\mathrm{M}$ animals had a score of only about 9 . After 35 days, $35 \%-\mathrm{M}$ animals presented scores of about 20 , whereas $35 \%-\mathrm{V}$ animals presented scores of about 17 with no complete toe clearance, tail down and trunk instability.

\section{Inclined plane test}

In the pretest, the angle of the inclined plane at which one rat could maintain itself for at least $5 \mathrm{~s}$ was about 85 degrees for all groups $\left(F_{2,28}=0.34, P=0.71\right)$. In the SO-V group there was no difference in average maximum angle at any time analyzed $\left(\mathrm{F}_{6,54}=1.20, \mathrm{P}=0.32\right)$. For the $35 \%-\mathrm{V}$ and $50 \%-\mathrm{V}$ groups, the average maximum angle decreased strikingly after narrowing and did not return to a normal level after 5 weeks $\left(F_{2,28}\right.$ ranging from 62.84 to $494.08, \mathrm{P}<0.0001)$. Furthermore, a $50 \%$ narrowing produced a more pronounced decrease in angle degree than a $35 \%$ narrowing at days $7,14,21,28$, and 35 post-injury $(\mathrm{P}<0.05)$.

Post-injury, the average maximum angle at which a rat could maintain itself on the inclined plane differed significantly between melatonin- and vehicle-treated rats in 35 and $50 \%$ animals (drug effect: $35 \%, F_{1,17}=10.03$, $\mathrm{P}=0.006 ; 50 \%, \mathrm{~F}_{1,20}=7.67, \mathrm{P}=0.012$, Figure 5). Melatonin-treated animals in the 35\%-M group showed higher mean angle degrees than $35 \%-\mathrm{V}$ animals at all time points post-injury ( $\mathrm{t}_{17}$ ranging from -3.58 to $-2.18, \mathrm{P}$ $<0.05)$. In 50\%-M rats the average maximum angle at which a rat could maintain itself on the inclined plane was detected at days 1,28 and 35 ( $\mathrm{t}_{20}$ ranging from -3.39 to $-0.78, \mathrm{P}<0.05)$

\section{Correlation between the BBB locomotor scale and the inclined plane test}

A strong correlation between BBB score and inclined 


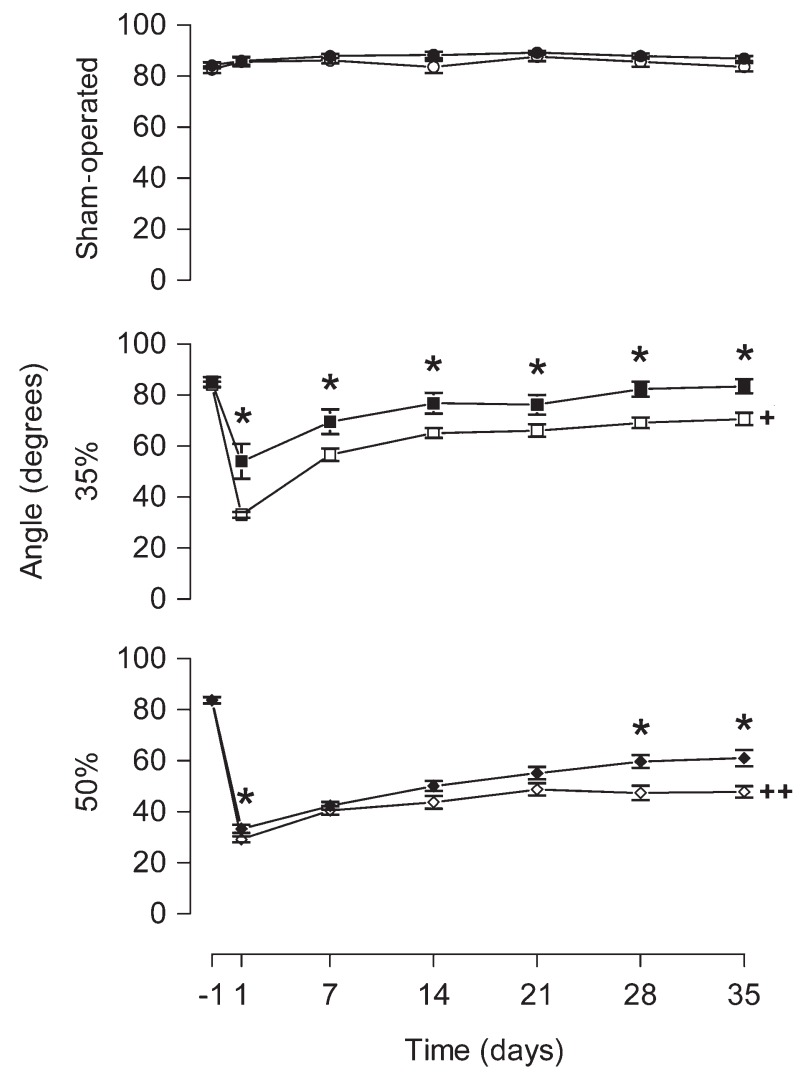

Figure 5. Melatonin treatment increased the angle on the inclined plane test. Results of the inclined plane test measured $24 \mathrm{~h}$ before (-1) and 1, 7, 14, 21, 28, and 35 days after narrowing of the spinal cord canal. Each point represents the mean \pm SE for 9-11 rats. ${ }^{*} P<0.05$ compared to the vehicle-treated group (independent Student $t$-test). ${ }^{+} \mathrm{P}<0.0001$ compared to sham-operated group from vehicle-treated animals, at all times after narrowing of the spinal cord canal (ANOVA with the Duncan post hoc test). ${ }^{+}{ }^{+} \mathrm{P}<0.0001$ compared to sham-operated group at all times after narrowing of the spinal cord canal and ${ }^{++} P<0.05$ compared to $35 \%$ narrowing, from vehicle-treated animals, on days $7,14,21,28$, and 35 after narrowing of the spinal cord canal (ANOVA with the Duncan post hoc test). 35\% = 35\% narrowing group; $50 \%=50 \%$ narrowing group. Open symbols $=$ vehicletreated groups; filled symbols = melatonin-treated groups.

plane angle degree was observed $\left(r=0.95, r^{2}=0.90, P\right.$ $<0.0001$ ). BBB scores of $0,7,14$, and 21 corresponded to approximately $23,44,65$, and 86 degrees on the inclined plane, respectively.

\section{NADPH-d histochemistry}

A photomicrograph of a typical spinal cord thoracic segment from a vehicle-treated animal, labeled with NADPH-d histochemistry is illustrated in Figure 6. Rats were sacrificed 35 days after sham operation (A-C) or $35 \%$ (D-F) or $50 \%$ (G-I) spinal cord narrowing. NADPH-d histochemistry revealed integrity of the labeled neurons $(\mathrm{A}-\mathrm{C})$ in the intermediolateral nucleus and lamina 10 (motoneurons). Photomicrographs in Panels D-I confirm the severity of the spinal cord lesion. Analysis of NADPH$\mathrm{d}$ activity in the spinal cords of the experimental groups showed that the density of labeled neurons varied with percent compression and with time (compression effect: $F_{2,160}=144.34, P<0.0001$; time effect: $F_{4,160}=3.95, P$ $=0.004$ ) but not with melatonin treatment (drug effect: $\left.F_{1,160}=0.77, P=0.38\right)$. Six to $48 \mathrm{~h}$ after both rostral and caudal spinal cord compression (0.0 to $0.39 \mathrm{~mm}$ for both sides) in the $35 \%-\mathrm{V}$ and $50 \%-\mathrm{V}$ groups, the density of NADPH-d-positive neurons decreased compared to SO-V animals in all Rexed laminae $\left(F_{2,15}\right.$ ranging from 4.69 to 38.63, $\mathrm{P}<0.03$ ). Moreover, after $24 \mathrm{~h}$, the density of NADPH-d-positive neurons observed in the laminae I-IV of $50 \%-\mathrm{V}$ animals, caudal segment, was lower than in $35 \%-\mathrm{V}$ animals. This was also observed for laminae III and IV after $48 \mathrm{~h}$, and for laminae I to IV and the intermediolateral nucleus after 35 days (Duncan post hoc test, $\mathrm{P}<0.05)$. The data for vehicle-treated animals, $24 \mathrm{~h}$ after surgery, are shown in Table 1.

\section{Discussion}

Based on behavioral and histological analyses, the present study showed that melatonin administration significantly improved functional recuperation after $\mathrm{SCI}$, extending previous results (9) obtained by the analysis of narrowing of the spinal cord canal and BBB locomotor score.

The hormone melatonin participates in many physiologic functions including regulation of circadian rhythms (16). More recently, melatonin was found to be a highly efficient scavenger of hydroxyl and peroxyl radicals as well as an electron donor $(4,7,16)$. Being lipophilic and hydrophilic, it has an extreme diffusibility for scavenging in cells and in every subcellular compartment. The efficacy of melatonin has been tested in many neurological disease models where free radicals have been pointed out as a pathologic causal factor. Thus, melatonin has been associated with neuroprotective effects in Alzheimer's and Parkinson's diseases, glutamate excitotoxicity and ischemia-reperfusion injury, amongst others (17). Although the primary protective effect of melatonin can be related to its ubiquitous direct and indirect antioxidant actions, other beneficial functions of melatonin are not precluded (4). Compared to methylprednisolone, melatonin exerts considerable neuroprotection, with the most prominent protective effect on myelin, nuclear axons and mitochondria (3). There is strong evidence that this hormone could be a powerful tool to prevent the secondary wave of neuronal death after SCI.

Narrowing of the spinal cord canal, as described by Dimar et al. (9), is an easy and suitable method for the study of $\mathrm{SCl}$, which produces gradual narrowing of the spinal cord canal resulting in progressive functional impairment and histological damage directly related to 

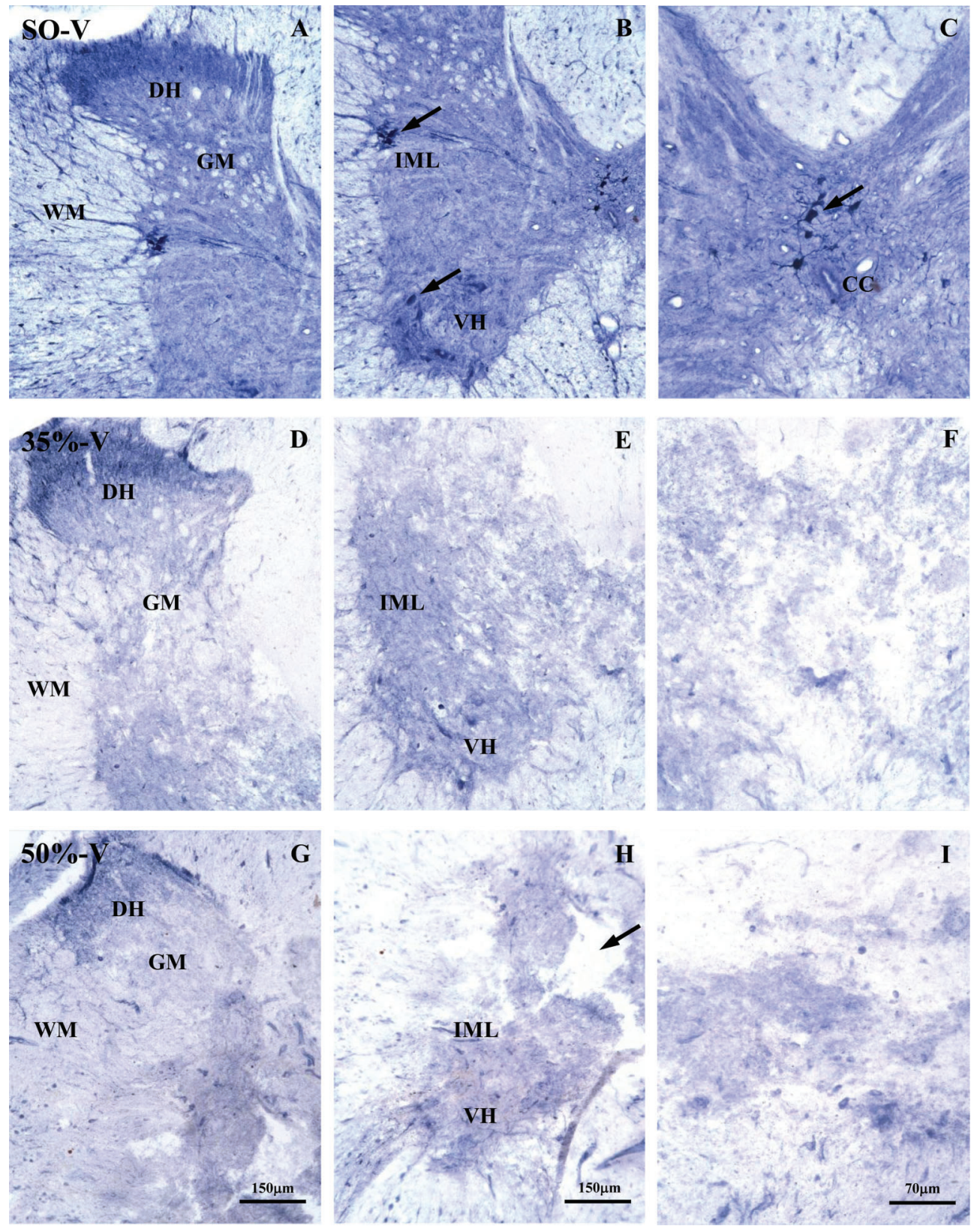

Figure 6. Photomicrograph of a typical thoracic spinal cord segment labeled by NADPH-d activity 35 days after the experimental procedure for the sham-operated + vehicle (SO-V) group $(A, B$ and $C)$, the narrowing of $35 \%+$ vehicle $(35 \%-V)(D, E$ and $F)$ group and the narrowing of $50 \%+$ vehicle $(50 \%-\mathrm{V})(G, H$ and $I)$ group. $D-/$ photomicrographs confirmed the severity of the spinal cord lesion. Arrows in $B$ highlight NADPH-d-positive neurons in the intermediolateral nucleus and in the ventral horn from gray matter. The arrow in $C$ highlights NADPH-d-positive neurons in lamina $X$ of Rexed from gray matter. The arrow in $H$ shows formation of a cavity near the central part of the spinal cord between white and gray matter. $\mathrm{DH}=$ dorsal horn; $\mathrm{GM}=$ gray matter; $\mathrm{VH}=$ ventral horn; $\mathrm{WM}=$ white matter; IML = intermediolateral nucleus; CC = central canal.

the degree of narrowing (9).

Laboratory rodents perform a wide variety of behaviors, many of which are relevant to assessing the integrity of spinal pathways. Different kinds of measurements can be potentially used to assess behavioral recovery after $\mathrm{SCl}$ in rats. A combination of functional tests for each animal has been recommended to provide a quantifiable and comprehensive analysis of sensorimotor function in spinal-injured rodents $(18,19)$. According to Muir and Weeb (19), a comprehensive analysis should 
Table 1. Density of NADPH-d-positive neurons in the Rexed laminae according to experimental group and segment in relation to the compression site.

\begin{tabular}{|c|c|c|c|c|c|}
\hline \multirow[t]{2}{*}{ Segment/group } & \multicolumn{5}{|c|}{ NADPH-d-positive neurons/50,000 $\mu \mathrm{m}^{2}$ (Rexed laminae) } \\
\hline & I and II & III and IV & IML & VIII and IX & $x$ \\
\hline \multicolumn{6}{|c|}{$0.0-0.39 \mathrm{~mm}$ (rostral) } \\
\hline SO-V & $31.30 \pm 2.06$ & $14.50 \pm 1.41$ & $29.17 \pm 4.23$ & $2.92 \pm 0.50$ & $5.99 \pm 1.45$ \\
\hline $35 \%-V$ & $9.65 \pm 3.19$ & $3.05 \pm 1.85$ & $1.72 \pm 1.72$ & $0.97 \pm 0.45$ & $0.00 \pm 0.00$ \\
\hline $50 \%-V$ & $11.33 \pm 2.40$ & $2.21 \pm 0.97$ & $0.00 \pm 0.00$ & $1.62 \pm 0.37$ & $1.00 \pm 0.74$ \\
\hline \multicolumn{6}{|c|}{$0.0-0.39 \mathrm{~mm}$ (caudal) } \\
\hline SO-V & $31.64 \pm 2.09$ & $11.45 \pm 1.41$ & $25.74 \pm 2.47$ & $3.41 \pm 0.47$ & $6.74 \pm 1.00$ \\
\hline $35 \%-V$ & $15.95 \pm 5.11$ & $5.43 \pm 2.91$ & $8.01 \pm 5.72$ & $1.22 \pm 0.64$ & $1.00 \pm 1.00$ \\
\hline $50 \%-V$ & $8.56 \pm 3.54$ & $0.51 \pm 0.26$ & $0.00 \pm 0.00$ & $0.08 \pm 0.08$ & $0.00 \pm 0.00$ \\
\hline
\end{tabular}

Data are reported as means \pm SE. I and II = superficial lamina; III and IV = nucleus proprius; IML = intermediolateral nucleus; VIII and IX = ventral horn; $X=$ region around the central canal; SO-V $=$ sham-operated + vehicle; $35 \%-V=$ narrowing of $35 \%+$ vehicle; $50 \%-\mathrm{V}=$ narrowing of $50 \%+$ vehicle.

include each of the following three components: i) an assessment of reflex function; ii) a measure of motor abilities during spontaneous locomotor activity, and iii) a measure of abilities during one or more trained behavioral tasks. Measurement of reflex function provides an indication of integrity of a particular spinal pathway. Spontaneous movement provides an initial screening of overall motor abilities. Finally, assessing the performance of trained behaviors eliminates differences in motivation between animals and provides more detailed and specific measures of functional abilities.

Locomotion over a flat surface is a behavior that involves the whole body. Rearing on the hind limbs involves primarily individual limbs (20). While just qualitative observations of locomotion are not sufficiently sensitive to assess the pattern of locomotion deficit (18), exploratory activity, rearing and grooming events are sensitive parameters for the detection of sensorimotor deficiencies (10). In summary, the results of the present study indicate that spinal cord injury produces a decrease in spontaneous locomotor activity as measured in an open-field test in spite of melatonin treatment. One day after surgery all animals, including the sham-operated ones, presented a decrease in ambulation, which, however, was more evident in the animals that suffered spinal cord compression. The decrease of ambulation by sham-operated animals can be explained by the repeated exposure to the open field with the novelty factor being pulled out. These results are in agreement with previous ones from Metz et al. (21) and Giglio et al. (10).

The BBB scale was designed to assess recovery of hind limb function after SCI (11). Each point in the score represents a specific set of characteristics demonstrated by the animal during spontaneous open-field locomotion. Although the BBB scale uses ordinal scores (22), the use of parametric statistics to evaluate its results has been more recently recommended. This would facilitate interpretation of the results between laboratories and provide a common methodology for behavioral data (23). In addition, Metz et al. (21) found that animals with low locomotion capacity presented a closer relationship between the BBB scale (11) and activity in the open field. In the present study, the BBB scale was able to consistently identify functional impairment after $\mathrm{SCl}$. Furthermore, by this method it was possible to differentiate the locomotor outcomes produced by gradual narrowing of the spinal cord canal and to show the progressive functional recuperation after injury.

The BBB scoring system correlated highly with the inclined plane test. When animals were assessed on the inclined plane the same impairment profile was observed, as also reported by Fujimoto et al. (6) after compression ischemia-induced $\mathrm{SCl}$. Although the inclined plane is a test where the quality of movement is not considered by this traditional, easy and quick method (12), it was possible to identify and differentiate functional deficits caused by the gradual narrowing of the spinal cord canal almost to the same extent as observed with the BBB score, a more comprehensive and detailed test. On the first day after injury, assessment of the animals on the inclined plane test was not able to show a difference between the $35 \%-V$ and $50 \%-V$ groups, whereas the difference was evident when using the BBB score system. Our results suggest that the inclined plane can be a reliable and easy test to be used together with other methods of behavior assessment to provide a complete overview of the motor behavior of the animal, helping to highlight discrete recovery after $\mathrm{SCl}$.

The neuroprotective effects of melatonin were confirmed in our study. These beneficial behavioral changes were more evident when animals were assessed by the BBB scale and by the inclined plane test. In agreement with the aforementioned findings, these methods were more reliable than others in highlighting the functional 
impairments caused by narrowing of the spinal cord canal after SCl. The preventive effect of melatonin was evident in $35 \%-M$ animals as early as on the first day post-injury, with melatonin-treated animals showing higher BBB scores and angle degrees on the inclined plane than vehicle-treated ones, suggesting that functional impairment was prevented by drug treatment early after the experimental procedure.

The progression of traumatic central nervous system injuries follows an archetypal course through primary and secondary damaging events, which are distinct in their spatiotemporal windows. There is a consensus that the best time window to use melatonin for a neuroprotective effect is just after the $\mathrm{SCl}$ event, using a single dose $(3,7,24)$ or multiple doses (6). Although there is no consensus regarding the use of single- or multiple-dose schemes, most of these studies have shown protective effects regarding both histological and behavioral aspects. In this study, we used five doses of melatonin in the acute window of $4 \mathrm{~h}$ as previously described by Fujimoto et al. (6), which were sufficient to improve the behavioral outcomes.

Our hypothesis is that the improvement in motor behavior observed in melatonin-treated animals after SCI is related to the properties of melatonin that prevent the secondary wave of neuronal death following the primary one after $\mathrm{SCl}$, including reactive astrogliosis (24). This may result from the inhibition of cysteine proteases (24), scavenging of hydroxyl and peroxyl radicals $(4,7,16)$, inhibition of nitric oxide synthase (NOS) activity $(7,16,25,26)$, or yet by other mechanisms that are not completely understood. Melatonin is supposed to prevent astrogliosis following acute $\mathrm{SCl}$ as demonstrated by Samantaray et al. (24) in a study in which a single dose of melatonin after weight-drop $\mathrm{SCl}$ attenuated the degree of astrocytosis in the penumbra, decreasing calpain and glial fibrillary acidic protein levels to those seen in vehicletreated animals $48 \mathrm{~h}$ after injury.

Melatonin has also been shown to inhibit NOS activity in vitro and in vivo $(7,16,25,26)$. Melatonin application decreased NO production in cultured cells (27), in brain homogenates (28) and after global or focal brain injury following ischemia/reperfusion $(29,30)$. Moreover, cerebral lipid peroxidation induced by NO donors was reduced

\section{References}

1. Mautes AE, Weinzierl MR, Donovan F, Noble LJ. Vascular events after spinal cord injury: contribution to secondary pathogenesis. Phys Ther 2000; 80: 673-687.

2. Christie SD, Comeau B, Myers T, Sadi D, Purdy M, Mendez I. Duration of lipid peroxidation after acute spinal cord injury in rats and the effect of methylprednisolone. Neurosurg Focus 2008; 25: E5, doi: 10.3171/FOC.2008.25.11.E5.

3. Kaptanoglu E, Tuncel M, Palaoglu S, Konan A, Demirpence E, Kilinc K. Comparison of the effects of melatonin and by melatonin treatment (31). NO is implicated in an enormous number of biological functions both in physiological and pathological conditions (32). As a free radical, $\mathrm{NO}$ seems to play a major role in the secondary lesion after acute spinal cord injury (33). The increased formation of NO, peroxynitrite and nitrated proteins shortly after spinal cord contusion (34) must play an important role in the destructive and reparative events taking place following acute spinal cord injury, depending on the amount of $\mathrm{NO}$ produced (35) and the redox conditions of the environment (36), among other factors. Hu et al. (37) showed a time-dependent differential increase in constitutive NOS and inducible NOS after lesion produced by dynorphin microinjection to the rat spinal cord. The same was observed after contusive (38) and compressive (39) spinal cord injury.

In our study, NADPH-d-positive neurons decreased in the rostral and caudal regions near the compression site after $\mathrm{SCl}$ in spite of melatonin treatment. Moreover, this decrease was mainly observed in the dorsal Rexed laminae (I-V) and more evident in the groups subjected to $50 \%$ compression. These results can be a reflex of the general neuronal loss after acute $\mathrm{SCl}$ and do not seem to explain the behavioral outcome observed in the melatonin-treated animals.

Our results confirm that melatonin acts as a neuroprotective agent after traumatic compression of the spinal cord. This may support the importance of beginning the neuroprotective treatment within the first 3-h period after $\mathrm{SCl}$ as reported in NASCIS III (40). These findings encourage further investigation of the potential role of melatonin in the neuroprotective process after injury.

\section{Acknowledgments}

The authors are indebted to Luciana N. Nascimento for skillful assistance during the study and to Prof. F.S. Guimarães (Departamento de Farmacologia, FMRPUSP) for advice concerning statistical analysis and text revision. This article was taken from the thesis for the PhD in Sciences of A. Schiaveto-de-Souza. Research supported by CNPq, FAPESP, and Don Bosco Universidade, Mato Grosso do Sul (A. Schiaveto-deSouza fellowship). methylprednisolone in experimental spinal cord injury. $J$ Neurosurg 2000; 93: 77-84, doi: 10.3171/ jns.2000.93.1.0077.

4. Reiter RJ, Korkmaz A. Clinical aspects of melatonin. Saudi Med J 2008; 29: 1537-1547.

5. Reiter R, Tang L, Garcia JJ, Munoz-Hoyos A. Pharmacological actions of melatonin in oxygen radical pathophysiology. Life Sci 1997; 60: 2255-2271, doi: 10.1016/S0024-3205(97)00030-1. 
6. Fujimoto T, Nakamura T, Ikeda T, Takagi K. Potent protective effects of melatonin on experimental spinal cord injury. Spine 2000; 25: 769-775, doi: 10.1097/00007632200004010-00003.

7. Topsakal C, Kilic N, Ozveren F, Akdemir I, Kaplan M, Tiftikci $M$, et al. Effects of prostaglandin E1, melatonin, and oxytetracycline on lipid peroxidation, antioxidant defense system, paraoxonase (PON1) activities, and homocysteine levels in an animal model of spinal cord injury. Spine 2003; 28: $1643-1652$.

8. Guízar-Sahagún G, Rodriguez-Balderas CA, FrancoBourland RE, Martinez-Cruz A, Grijalva I, Ibarra A, et al. Lack of neuroprotection with pharmacological pretreatment in a paradigm for anticipated spinal cord lesions. Spinal Cord 2009; 47: 156-160, doi: 10.1038/sc.2008.85.

9. Dimar JR, Glassman SD, Raque GH, Zhang YP, Shields $\mathrm{CB}$. The influence of spinal canal narrowing and timing of decompression on neurologic recovery after spinal cord contusion in a rat model. Spine 1999; 24: 1623-1633, doi: 10.1097/00007632-199908150-00002.

10. Giglio CA, Defino HL, da-Silva CA, de-Souza AS, Del Bel EA. Behavioral and physiological methods for early quantitative assessment of spinal cord injury and prognosis in rats. Braz J Med Biol Res 2006; 39: 1613-1623, doi: 10.1590/ S0100-879X2006001200013.

11. Basso DM, Beattie MS, Bresnahan JC. A sensitive and reliable locomotor rating scale for open field testing in rats. $J$ Neurotrauma 1995; 12: 1-21, doi: 10.1089/neu.1995.12.1.

12. Rivlin AS, Tator $\mathrm{CH}$. Objective clinical assessment of motor function after experimental spinal cord injury in the rat. $J$ Neurosurg 1977; 47:577-581, doi: 10.3171/jns.1977.47.4.0577.

13. Paxinos G, Watson $\mathrm{C}$. The rat brain in stereotaxic coordinates. Burlington: Academic Press; 2005.

14. Vincent SR, Kimura $\mathrm{H}$. Histochemical mapping of nitric oxide synthase in the rat brain. Neuroscience 1992; 46: 755784, doi: 10.1016/0306-4522(92)90184-4.

15. Rogerio F, de Souza QL, Teixeira SA, Oliveira AL, de Nucci G, Langone F. Neuroprotective action of melatonin on neonatal rat motoneurons after sciatic nerve transection. Brain Res 2002; 926: 33-41, doi: 10.1016/S0006-8993(01)03286-3.

16. Cheung RT. The utility of melatonin in reducing cerebral damage resulting from ischemia and reperfusion. $J$ Pineal $R e s$ 2003; 34: 153-160, doi: 10.1034/j.1600-079X.2003.00034.x.

17. Reiter RJ. Oxidative damage in the central nervous system: protection by melatonin. Prog Neurobiol 1998; 56: 359-384, doi: 10.1016/S0301-0082(98)00052-5.

18. Kunkel-Bagden E, Dai HN, Bregman BS. Methods to assess the development and recovery of locomotor function after spinal cord injury in rats. Exp Neurol 1993; 119: 153-164, doi: 10.1006/exnr.1993.1017.

19. Muir GD, Webb AA. Mini-review: assessment of behavioural recovery following spinal cord injury in rats. Eur $J$ Neurosci 2000; 12: 3079-3086, doi: 10.1046/j.1460-9568.2000.00205.x.

20. Ramon-Cueto A, Avila J. Two modes of microtubuleassociated protein 1B phosphorylation are differentially regulated during peripheral nerve regeneration. Brain Res 1999; 815: 213-226, doi: 10.1016/S0006-8993(98)01092-0.

21. Metz GA, Merkler D, Dietz V, Schwab ME, Fouad K. Efficient testing of motor function in spinal cord injured rats. Brain Res 2000; 883: 165-177, doi: 10.1016/S00068993(00)02778-5.
22. Kerlinger $F$, Rokeach $M$. The factorial nature of the $F$ and $D$ scales. J Pers Soc Psychol 1966; 4: 391-399, doi: 10.1037/ h0023731.

23. Scheff SW, Saucier DA, Cain ME. A statistical method for analyzing rating scale data: the BBB locomotor score. $J$ Neurotrauma 2002; 19: 1251-1260, doi: 10.1089/ 08977150260338038.

24. Samantaray S, Sribnick EA, Das A, Knaryan VH, Matzelle DD, Yallapragada AV, et al. Melatonin attenuates calpain upregulation, axonal damage and neuronal death in spinal cord injury in rats. J Pineal Res 2008; 44: 348-357, doi: 10.1111/j.1600-079X.2007.00534.x.

25. Reiter RJ, Tan DX, Qi WB. Suppression of oxygen toxicity by melatonin. Zhongguo Yao Li Xue Bao 1998; 19: 575-581.

26. Reiter RJ, Tan DX, Osuna C, Gitto E. Actions of melatonin in the reduction of oxidative stress. A review. J Biomed Sci 2000; 7: 444-458, doi: 10.1007/BF02253360.

27. Lahiri DK, Ghosh C. Interactions between melatonin, reactive oxygen species, and nitric oxide. Ann N Y Acad Sci 1999; 893: 325-330, doi: 10.1111/j.1749-6632.1999.tb07847.x.

28. Teixeira A, Morfim MP, de Cordova CA, Charao CC, de Lima V, Creczynski-Pasa TB. Melatonin protects against pro-oxidant enzymes and reduces lipid peroxidation in distinct membranes induced by the hydroxyl and ascorbyl radicals and by peroxynitrite. J Pineal Res 2003; 35: 262268, doi: 10.1034/j.1600-079X.2003.00085.x.

29. Pei Z, Ho HT, Cheung RT. Pre-treatment with melatonin reduces volume of cerebral infarction in a permanent middle cerebral artery occlusion stroke model in the rat. Neurosci Lett 2002; 318: 141-144, doi: 10.1016/S0304-3940(01)02503-4.

30. Pei Z, Fung PC, Cheung RT. Melatonin reduces nitric oxide level during ischemia but not blood-brain barrier breakdown during reperfusion in a rat middle cerebral artery occlusion stroke model. J Pineal Res 2003; 34: 110-118, doi: 10.1034/ j.1600-079X.2003.00014.X.

31. Escames G, Guerrero JM, Reiter RJ, Garcia JJ, MunozHoyos A, Ortiz GG, et al. Melatonin and vitamin E limit nitric oxide-induced lipid peroxidation in rat brain homogenates. Neurosci Lett 1997; 230: 147-150, doi: 10.1016/S03043940(97)00498-9.

32. Pagliaro P. Differential biological effects of products of nitric oxide (NO) synthase: it is not enough to say NO. Life Sci 2003; 73: 2137-2149, doi: 10.1016/S0024-3205(03)00593-9.

33. Amar AP, Levy ML. Pathogenesis and pharmacological strategies for mitigating secondary damage in acute spinal cord injury. Neurosurgery 1999; 44: 1027-1039, doi: 10.1097/00006123-199905000-00052.

34. Liu D, Ling X, Wen J, Liu J. The role of reactive nitrogen species in secondary spinal cord injury: formation of nitric oxide, peroxynitrite, and nitrated protein. $J$ Neurochem 2000; 75: 2144-2154, doi: 10.1046/j.1471-4159.2000.0752144.x.

35. Kroncke KD, Fehsel K, Kolb-Bachofen V. Nitric oxide: cytotoxicity versus cytoprotection - how, why, when, and where? Nitric Oxide 1997; 1: 107-120, doi: 10.1006/niox.1997.0118.

36. Xu M, Ng YK, Leong SK. Neuroprotective and neurodestructive functions of nitric oxide after spinal cord hemisection. Exp Neurol 2000; 161: 472-480, doi: 10.1006/exnr.1999.7278.

37. Hu WH, Qiang WA, Li F, Liu N, Wang GQ, Wang HY, et al. Constitutive and inducible nitric oxide synthases after dynorphin-induced spinal cord injury. $J$ Chem Neuroanat 2000; 17: 183-197, doi: 10.1016/S0891-0618(99)00039-3. 
38. Diaz-Ruiz A, Ibarra A, Perez-Severiano F, Guizar-Sahagun G, Grijalva I, Rios C. Constitutive and inducible nitric oxide synthase activities after spinal cord contusion in rats. Neurosci Lett 2002; 319: 129-132, doi: 10.1016/S03043940(01)02540-X.

39. Nakahara S, Yone K, Setoguchi T, Yamaura I, Arishima Y, Yoshino S, et al. Changes in nitric oxide and expression of nitric oxide synthase in spinal cord after acute traumatic injury in rats. J Neurotrauma 2002; 19: 1467-1474, doi: 10.1089/089771502320914697.

40. Bracken MB, Shepard MJ, Holford TR, Leo-Summers L, Aldrich EF, Fazl M, et al. Methylprednisolone or tirilazad mesylate administration after acute spinal cord injury: 1year follow up. Results of the third National Acute Spinal Cord Injury randomized controlled trial. J Neurosurg 1998; 89: 699-706, doi: 10.3171/jns.1998.89.5.0699. 